\title{
Determinants of the impact of blood pressure variability on neurological outcome after acute ischaemic stroke
}

\author{
Adam de Havenon, ${ }^{1}$ Alicia Bennett, ${ }^{2}$ Gregory J Stoddard, ${ }^{2}$ Gordon Smith, ${ }^{2}$ \\ Lee Chung, ${ }^{2}$ Steve O'Donnell, ${ }^{2} \mathrm{~J}$ Scott McNally, ${ }^{2}$ David Tirschwell, ${ }^{3}$ \\ Jennifer J Majersik ${ }^{2}$
}

To cite: de Havenon A, Bennett A, Stoddard GJ, et al. Determinants of the impact of blood pressure variability on neurological outcome after acute ischaemic stroke. Stroke and Vascular Neurology 2017;2:e000057. doi:10.1136/svn-2016000057

Received 18 November 2016 Accepted 9 December 2016 Published Online First 27 January 2017

\section{CrossMark}

\footnotetext{
${ }^{1}$ Department of Neurology, University of Utah, Salt Lake City, Utah, USA

${ }^{2}$ University of Utah, Salt Lake City, Utah, USA

${ }^{3}$ University of Washington, Seattle, Washington, USA
}

Correspondence to Dr Adam de Havenon; adam.dehavenon@hsc. utah.edu

\section{ABSTRACT}

Introduction: Increased blood pressure variability (BPV) is detrimental after acute ischaemic stroke, but the interaction between BPV and neuroimaging factors that directly influence stroke outcome has not been explored.

Methods: We retrospectively reviewed inpatients from 2007 to 2014 with acute anterior circulation ischaemic stroke, CT perfusion and angiography at hospital admission, and a modified Rankin Scale (mRS) 30365 days after stroke onset. BPV indices included SD, coefficient of variation and successive variation of the systolic blood pressure between 0 and 120 hours after admission. Ordinal logistic regression models were fitted to $\mathrm{mRS}$ with predictor variables of BPV indices. Models were further stratified by CT perfusion volumetric measurements, proximal vessel occlusion and collateral score.

Results: 110 patients met the inclusion criteria. The likelihood of a 1-point rise in the mRS increased with every $10 \mathrm{~mm} \mathrm{Hg}$ increase in BPV (OR for the $3 \mathrm{BPV}$ indices ranged from 2.27 to 5.54 ), which was more pronounced in patients with larger ischaemic core volumes (OR 8.37 to 18.0 ) and larger hypoperfused volumes (OR 6.02 to 15.4). This association also held true for patients with larger mismatch volume, proximal vessel occlusion and good collateral vessels. Conclusions: These results indicate that increased $\mathrm{BPV}$ is associated with worse neurological outcome after stroke, particularly in patients with a large lesion core volume, concurrent viable ischaemic penumbra, proximal vessel occlusion and good collaterals. This subset of patients, who are often not candidates for or fail acute stroke therapies such as intravenous tissue plasminogen activator or endovascular thrombectomy, may benefit from interventions aimed at reducing BPV.

\section{INTRODUCTION}

Increased blood pressure (BP) variability (BPV), independent of the BP mean, is harmful after ischaemic and haemorrhagic stroke. $^{1-7}$ Under normal circumstances, dynamic autoregulation of the cerebrovascular bed maintains a relatively constant cerebral blood flow (CBF) across a wide range of
BPs. ${ }^{8}{ }^{9}$ However, after ischaemic stroke, the ability to autoregulate is often impaired in the area of the lesion core and ischaemic penumbra. ${ }^{10} 11$ As a result, the penumbra can be directly exposed to deleterious fluctuations in systemic BP and increased BPV has been shown to result in lesion core growth on diffusion-weighted MRI 3648 hours post-stroke. ${ }^{12}$ Prior analyses of BPV have not evaluated the impact of admission lesion core volume or other characteristics of the ischaemic penumbra, which are important radiological predictors of clinical outcome and response to acute stroke treatments. ${ }^{13}{ }^{14}$ Additional neuroimaging determinants of outcome, such as proximal vessel occlusion (PVO) and cerebral collateral vessel status, have likewise not been evaluated in past BPV studies. ${ }^{89}$ To address these questions, we examined the impact of CT perfusion (CTP) volumetric measurements, PVO and collateral vessel status on the interaction between BPV and neurological outcome among a cohort of patients with acute ischaemic stroke.

\section{METHODS}

\section{Patient selection}

Patients were retrospectively identified by searching the electronic medical record of an academic medical centre for ischaemic stroke International Classification of Diseases (ICD)-9 codes between 2007 and 2014. Patients were included who had a CTP and angiographic imaging at hospital admission, an anterior circulation stroke confirmed by a neurologist, BP data available for 120 hours after admission and a follow-up mRS 30-365 days after stroke onset. If $\mathrm{mRS}$ was 0 (no symptoms) or 6 (death) at hospital discharge, it was carried forward as a follow-up mRS. Lacunar strokes were excluded because CTP imaging is not sensitive to small perfusion abnormalities. We 
selected the 120-hour interval for calculating BPV because the two largest studies of BPV included BP data for up to 7 days after stroke onset and many other studies focused on the first 72 hours after onset. ${ }^{15}$ The 120 -hour interval allowed us to include most patients while also acquiring a
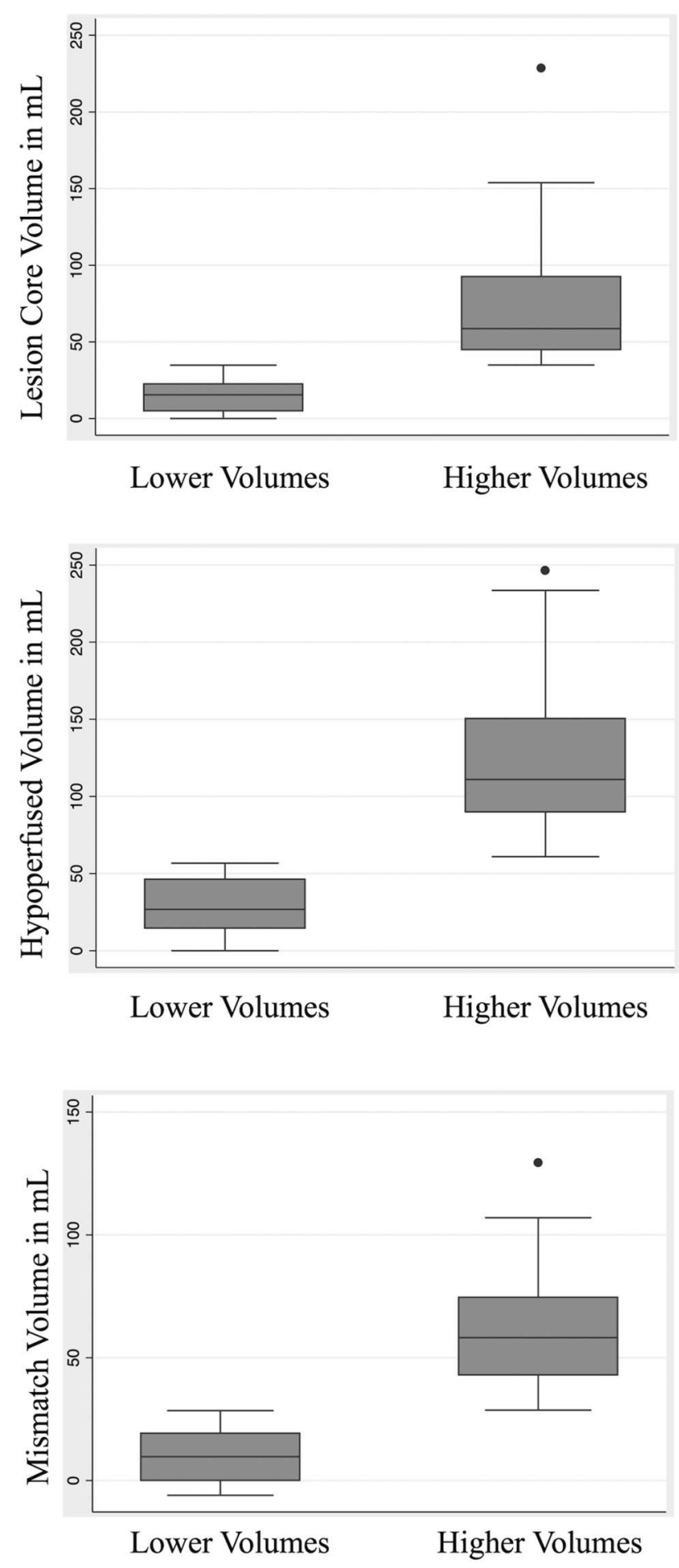

Figure 1 CT perfusion volumetric measurements shown for dichotomous stratifications of lesion core volume, hypoperfused volume, and mismatch volume with box plot representation of median line and IQR, whisker representation of data range, and outliers as single data points. sufficient number of BP readings per patient to reliably determine variability. Additional information was obtained from the chart, including admission National Institutes of Health (NIH) Stroke Scale (NIHSS), patient demographics, medical comorbidities, admission laboratory values, data from angiographic imaging, administration of intravenous tissue plasminogen activator (tPA) and performance of endovascular therapy (defined as mechanical or aspiration thrombectomy or intra-arterial tPA).

\section{Imaging parameters and analysis}

Symptomatic intracerebral haemorrhage (sICH) was identified on non-contrast head CT or MRI and defined using the European Cooperative Acute Stroke Study 2 criteria. $^{16}$ CTP was performed using a 64-section scanner (Definition or Definition AS; Siemens) using a four-dimensional spiral technique as previously described. ${ }^{17}$ Standard imaging parameters were $80 \mathrm{kVp}$, $200 \mathrm{mAs}, 4 \mathrm{~mm}$ slice thickness, $8.4 \mathrm{~cm}$ total coverage. Approximately $40 \mathrm{~mL}$ of non-ionic iodinated contrast was administered intravenously at $7 \mathrm{~mL} / \mathrm{s}$ using a power injector.

CTP source images were used to assess for the presence of cerebral collateral blood vessels (CTP collaterals) in the region of the Sylvian fissure and leptomeningeal convexity based on a validated ordinal scale. ${ }^{18}$ Collateral vessels were graded by comparing the symptomatic hemisphere to the contralateral hemisphere as follows: (1) absent; (2) less than the contralateral normal side; (3) equal to the contralateral normal side; (4) greater than the contralateral normal side. For both sICH and CTP collaterals, two experienced raters (AdH, JSM) graded a representative portion $(30 \%)$ of the cohort and the results were compared with two additional raters $(\mathrm{AB}, \mathrm{SO})$, who were allowed to continue grading the remainder of the cohort because their interrater reliability $(\kappa)$ with the experienced readers was $>0.9$. For statistical analysis, the cohort was stratified by good collaterals (CTP collateral score 3-4) versus bad collaterals (CTP collateral score 1-2). Further stratification was made by $\mathrm{PVO}$, which was defined as occlusion of the internal carotid artery or M1 segment of the middle cerebral artery on admission MR, CT or digital subtraction angiogram.

For volumetric analysis, we used the Food and Drug Administration (FDA)-approved Olea Sphere software (Olea Medical: La Ciotat, France) to generate CTP maps with a Bayesian-based probabilistic deconvolution method, which recent data suggest is superior to other delay-insensitive methods. ${ }^{19-21}$ On the basis of previously validated CTP threshold definitions, we defined a lesion core as relative $\mathrm{CBF}<40 \%$ and absolute arterial tissue delay $>2 \mathrm{~s}$, and hypoperfused tissue as relative mean transit time $>135 \%{ }^{19}{ }^{22} 23$ The CTP data were used to create dichotomous patient stratifications based on three volumetric categories (figure 1): upper and lower halves of lesion core volume, hypoperfused volume, and mismatch volume 
(hypoperfused-lesion core volume), which correspond to the concept of ischaemic penumbra. A fourth dichotomous stratification was made by the 'Target Mismatch' profile (hypoperfused:lesion ratio $>1.8$, mismatch volume $>15 \mathrm{~mL}$ and lesion core volume $<70 \mathrm{~mL}$ ), which has been validated for both MR perfusion and CTP. ${ }^{24} 25$

\section{Statistical analysis}

BPV was calculated using systolic BP (SBP) readings between 0 and 120 hours from hospital admission. Over $80 \%$ of patients had haemodynamic data starting within 6 hours of stroke onset and the remainder had it within 24 hours. Haemodynamic data that were considered non-physiological $\quad(\mathrm{SBP}>280$ or $\quad<50 \mathrm{~mm} \mathrm{Hg}) \quad$ were changed to missing, which was fewer than $0.05 \%$ of available measurements. BPV was calculated in three ways-SD: $\sqrt{(1 /(n-1)) \sum_{(\mathrm{i}=1)}^{(\mathrm{n})}\left(\mathrm{BP}_{\mathrm{i}}-\mathrm{BP}_{\text {mean }}\right)^{2}}$, coefficient of variation $(\mathrm{CV}(\%))$ : $\mathrm{SD} / \mathrm{BP}_{\text {mean }} \times 100$, and successive variation $(\mathrm{SV})$ calculated as the square root of the average difference in BP between successive measurements using the equation: $\sqrt{\left.(1 /(\mathrm{n}-1)) \sum_{(\mathrm{i}=1)}^{(\mathrm{n}-1)}\left(\mathrm{BP}_{\mathrm{i}+1}-\mathrm{BP}_{\mathrm{i}}\right)^{2}\right)^{6}}$ We choose SD, CV and SV based on prior literature suggesting that multiple approaches to measuring BPV should be employed. ${ }^{4}$

Stata V.14.1 was used for all data analyses, with statistical significance defined as $\mathrm{p}<0.05$. Intergroup differences were evaluated with Spearman's rank correlation, independent sample t-test, $\chi^{2}$ test and the Mann-Whitney $\mathrm{U}$ test. The regression analyses were calculated with ordinal logistic regression fitted to the outcome of mRS. This statistical methodology allows measurement of shift in mRS, the odds of moving to the next score, which is particularly beneficial when the effect of the intervention or clinical factor is spread across the entire range of ordinal values. ${ }^{26-28}$ An ordinal logistic regression model was fitted to the outcome of $\mathrm{mRS}$ with individual BPV indices. Multivariable ordinal regression models were fitted to control for possible confounders using an interactive backward variable selection (inclusion with $\mathrm{p}<0.05$ ). The ordinal logistic regression models were stratified by the four dichotomous categories of the upper and lower halves of lesion core volume, hypoperfused volume, mismatch volume and Target Mismatch. In keeping with recommendations from the recent meta-analysis on BPV, ORs and $95 \%$ CIs are reported per $10 \mathrm{~mm} \mathrm{Hg}$ increment in the BPV parameter. ${ }^{15}$ If $2 / 3$ of the BPV indices were significant for a given model, it was considered a relevant finding.

\section{RESULTS}

One hundred and ten patients met the inclusion criteria. Patient demographics are shown in table 1 . There were $6587 \mathrm{BP}$ readings between 0 and 120 hours after stroke onset and the median number of $\mathrm{BP}$ readings per patient was 57 (IQR 50-66). There were a high number of PVOs at hospital admission (58/110, 53\%). An additional 32/110 (29\%) had an M2 or A1 segment occlusion with the remainder of patients $(20 / 110,18 \%)$ having more distal M3 or A2 occlusions. Half of the patients were administered intravenous tPA and 40\% (44/110) had endovascular intervention, and 22\% (24/110) had both. The high number of acute stroke interventions is secondary to the referral pattern for CTP at our institution. A relatively high number of patients developed sICH $(13 / 110,11.8 \%)$, reflecting the increased risk for sICH with interventional stroke therapy and the high median NIHSS (12, IQR 7-19) in our cohort. The mean \pm SD lesion core and hypoperfused volumes were $43.8 \pm 40.6 \mathrm{~mL}$ and $75.9 \pm 56.9$, creating a moderate mismatch volume (hypoperfused-lesion volume) of $36.8 \pm 31.1 \mathrm{~mL}$. The median CTP collateral score was 3, but the most common value was $2(44 / 110,40 \%)$.

In the adjusted and unadjusted ordinal logistic regression models fitted to the outcome of mRS, all three measures of BPV (SBP CV, SD and SV) were predictive of a one-point shift in the mRS (OR 2.27 to 5.54, p<0.05; table 2). SBP mean was not predictive of outcome and

Table 1 Patient demographics, clinical information and initial imaging data

\begin{tabular}{|c|c|}
\hline Variable & $\begin{array}{l}\text { All patients } \\
(n=110)\end{array}$ \\
\hline Age, years, mean $\pm S D$ & $61.5 \pm 17.0$ \\
\hline Male, n (\%) & $60(55.6)$ \\
\hline Caucasian, n (\%) & $95(86.4)$ \\
\hline Admission NIHSS, median (IQR) & $12(7-19)$ \\
\hline Follow-up mRS, median (IQR) & $3(1-4)$ \\
\hline $\begin{array}{l}\text { Time to follow-up mRS from stroke, days, } \\
\text { mean } \pm S D\end{array}$ & $96 \pm 51$ \\
\hline Hypertension, n (\%) & $60(54.6)$ \\
\hline Hyperlipidaemia, n (\%) & $41(37.3)$ \\
\hline Atrial fibrillation, $\mathrm{n}(\%)$ & $33(30.0)$ \\
\hline Diabetes mellitus, $\mathrm{n}(\%)$ & $23(20.9)$ \\
\hline Congestive heart failure, $\mathrm{n}(\%)$ & $14(12.7)$ \\
\hline Current cigarette smoking, $\mathrm{n}(\%)$ & $24(21.8)$ \\
\hline Admission glucose level, mg/dL, mean \pm SD & $127.1 \pm 40.2$ \\
\hline Collateral score (1-4), median (IQR) & $3(2-4)$ \\
\hline Lesion volume, $\mathrm{mL}$, mean $\pm \mathrm{SD}$ & $43.8 \pm 40.6$ \\
\hline Hypoperfused volume, $\mathrm{mL}$, mean $\pm \mathrm{SD}$ & $75.9 \pm 56.9$ \\
\hline Mismatch volume, $\mathrm{mL}$, mean $\pm \mathrm{SD}$ & $36.8 \pm 31.1$ \\
\hline tPA administered, $\mathrm{n}(\%)$ & $55(50.0)$ \\
\hline Endovascular therapy, n (\%) & $44(40.0)$ \\
\hline $\begin{array}{l}\text { Symptomatic intracerebral haemorrhage, } \\
\mathrm{n}(\%)\end{array}$ & $13(11.8)$ \\
\hline Proximal vessel occlusion, n (\%) & $58(52.7)$ \\
\hline SBP SD, 0-120 hours, mean \pm SD & $14.4 \pm 4.8$ \\
\hline SBP CV, 0-120 hours, mean \pm SD & $11.1 \pm 3.7$ \\
\hline SBP SV, 0-120 hours, mean \pm SD & $14.1 \pm 4.5$ \\
\hline SBP mean, 0-120 hours, mean \pm SD & $131.0 \pm 16.7$ \\
\hline
\end{tabular}


Table 2 Unadjusted and adjusted ORs for a one-point shift in mRS at follow-up with predictor blood pressure indices of SBP $\mathrm{SD}, \mathrm{CV}, \mathrm{SV}$ and mean. ORs are shown for a $10 \mathrm{~mm} \mathrm{Hg}$ shift

\begin{tabular}{lllllll}
\hline Blood pressure indices & $\begin{array}{l}\text { OR for a 1-point } \\
\text { mRS shift }\end{array}$ & $\mathbf{9 5 \%}$ Cl & p Value & $\begin{array}{l}\text { Adjusted OR for } \\
\text { a 1-point } \text { mRS shift }^{*}\end{array}$ & $\mathbf{9 5 \%}$ Cl & p Value \\
\hline SBP CV & 3.30 & 1.48 to 7.35 & 0.003 & 3.02 & 0.86 to 10.6 & 0.085 \\
SBP SD & 5.54 & 1.72 to 17.9 & 0.004 & 2.78 & 1.16 to 6.70 & 0.022 \\
SBP SV & 2.27 & 1.01 to 5.10 & 0.047 & 3.03 & 1.28 to 7.17 & 0.012 \\
SBP mean & 1.00 & 0.98 to 1.02 & 0.722 & 1.02 & 1.00 to 1.05 & 0.038 \\
\hline
\end{tabular}

${ }^{*}$ Adjusted for admission NIHSS, patient sex, history of congestive heart failure, history of diabetes mellitus and symptomatic intracranial haemorrhage.

CV, coefficient of variation; mRS, modified Rankin Scale; NIHSS, National Institutes of Health Stroke Scale; SBP, systolic blood pressure; SV, successive variation.

hence was not included in subsequent models. In unadjusted ordinal models, the CTP dichotomous stratifications demonstrated an association between increased $\mathrm{BPV}$ and worse outcome in patients with larger lesion core volume (OR 8.37 to $18.0, \mathrm{p}<0.05$ ), larger hypoperfused volume (OR 6.02 to $15.4, \mathrm{p}<0.05$ ) and mismatch volume (OR 3.66 to $9.41, \mathrm{p}<0.05$ ), but the association was not significant in the lower halves of the stratifications. These relationships maintained significance after adjusting for possible confounders, including admission NIHSS, patient sex, tPA administration, sICH and admission glucose (table 3).

Additional stratifications were made based on the Target Mismatch profile, PVO at hospital admission and collateral score. In the unadjusted model, patients without Target Mismatch had an association between increased $\mathrm{BPV}$ and worse neurological outcome (OR 5.26 to $8.43, \mathrm{p}<0.05$ ), which continued to be significant in the adjusted model (table 4). Patients with PVO and good collaterals also demonstrated an association between increased BPV and worse outcome (OR 5.20 to $9.60,3.58$ to $31.9, \mathrm{p}<0.05)$. These associations also remained significant in the adjusted models (table 4 ).

\section{DISCUSSION}

Our results confirm earlier reports that increased BPV is harmful after acute ischaemic stroke $\mathrm{e}^{1-7}$ and the inclusion of stratifications based on neuroimaging determinants such as CTP volumetric data, PVO and cerebral collateral status adds a novel perspective. These analyses revealed that patients with larger ischaemic core or hypoperfused volumes are particularly vulnerable to the detrimental effects of increased BPV. This relationship was also seen in patients with a larger mismatch and without the Target Mismatch profile. Taken together, these findings suggest that the impact of increased BPV is, at its most fundamental level, driven by the larger absolute volumes of infarcted and peri-infarct tissue.

Increased BPV has been linked to the development of sICH after ischaemic stroke, ${ }^{29}$ which would be one plausible mechanism for why patients with larger core and hypoperfused volumes had a worse outcome with higher BPV, but the incidence of sICH was not different in any of the stratifications and it was included as a covariate in the adjusted models. A more compelling explanation is that after moderate-to-severe ischaemic stroke, the lesion core and its ischaemic penumbra often

Table 3 Adjusted ORs for a one-point shift in mRS at follow-up with predictor variables of SBP SD, CV and SV; stratified by lesion core volume, hypoperfused volume, mismatch volume, Target Mismatch status, proximal vessel occlusion on admission and collateral score

\begin{tabular}{|c|c|c|c|c|c|c|c|}
\hline BPV indices & OR $^{*}$ & $95 \% \mathrm{Cl}$ & p Value & BPV indices & OR $^{*}$ & $95 \% \mathrm{Cl}$ & p Value \\
\hline \multicolumn{4}{|c|}{ Higher lesion core volume $(n=55)($ mean $\pm S D=72.7 \pm 39.2 \mathrm{~mL})$} & \multicolumn{4}{|c|}{ Lower lesion core volume $(n=55)($ mean $\pm S D=15.1 \pm 10.3 \mathrm{~mL})$} \\
\hline SBP SD & 9.27 & 2.36 to 36.3 & 0.001 & SBP SD & 0.74 & 0.21 to 2.63 & 0.643 \\
\hline SBP CV & 20.2 & 3.00 to 137 & 0.002 & SBP CV & 0.30 & 0.05 to 2.07 & 0.224 \\
\hline SBP SV & 18.9 & 3.69 to 97.1 & $<0.001$ & SBP SV & 1.27 & 0.44 to 3.66 & 0.664 \\
\hline \multicolumn{4}{|c|}{ Higher hypoperfused volume $(n=55)($ mean $\pm S D=121.3$} & \multicolumn{4}{|c|}{$\begin{array}{l}\text { Lower hypoperfused volume }(n=55)(\text { mean } \pm S D=30.5 \\
\pm 17.6 \mathrm{~mL})\end{array}$} \\
\hline SBP SD & 5.41 & 1.24 to 23.6 & 0.025 & SBP SD & 0.85 & 0.23 to 3.10 & 0.804 \\
\hline SBP CV & 12.9 & 1.70 to 98.8 & 0.013 & SBP CV & 0.28 & 0.04 to 2.01 & 0.204 \\
\hline SBP SV & 4.09 & 0.99 to 16.9 & 0.052 & SBP SV & 1.63 & 0.52 to 5.08 & 0.402 \\
\hline \multicolumn{4}{|c|}{ Higher mismatch volume $(n=55)($ mean $\pm S D=62.3 \pm 22.9 \mathrm{~mL})$} & \multicolumn{4}{|c|}{ Lower mismatch volume $(n=55)($ mean $\pm S D=11.4 \pm 10.4 \mathrm{~mL})$} \\
\hline SBP SD & 3.35 & 1.03 to 11.0 & 0.045 & SBP SD & 2.58 & 0.58 to 11.4 & 0.212 \\
\hline SBP CV & 5.97 & 1.05 to 34.0 & 0.044 & SBP CV & 1.24 & 0.16 to 9.36 & 0.838 \\
\hline SBP SV & 3.76 & 1.13 to 12.5 & 0.031 & SBP SV & 2.44 & 0.61 to 9.87 & 0.210 \\
\hline
\end{tabular}


Table 4 Adjusted ORs for a one-point shift in mRS at follow-up with predictor variables of SBP SD, CV and SV; stratified by lesion core volume, hypoperfused volume, mismatch volume, Target Mismatch status, proximal vessel occlusion on admission and collateral score

\begin{tabular}{|c|c|c|c|c|c|c|c|}
\hline BPV indices & $\mathbf{O R}^{*}$ & $95 \% \mathrm{Cl}$ & p Value & BPV indices & OR $^{*}$ & $95 \% \mathrm{Cl}$ & p Value \\
\hline \multicolumn{4}{|c|}{ Target Mismatch $(n=57)$} & \multicolumn{4}{|c|}{ No Target Mismatch $(n=53)$} \\
\hline SBP SD & 1.94 & 0.63 to 6.02 & 0.250 & SBP SD & 6.61 & 1.40 to 31.1 & 0.017 \\
\hline SBP CV & 2.39 & 0.45 to 12.7 & 0.305 & SBP CV & 5.32 & 0.70 to 40.1 & 0.105 \\
\hline SBP SV & 2.56 & 0.81 to 8.09 & 0.109 & SBP SV & 5.96 & 1.20 to 29.6 & 0.029 \\
\hline \multicolumn{4}{|c|}{ Proximal vessel occlusion $(n=58)$} & \multicolumn{4}{|c|}{ No proximal occlusion $(n=52)$} \\
\hline SBP SD & 5.38 & 1.44 to 20.2 & 0.013 & SBP SD & 1.63 & 0.53 to 5.03 & 0.398 \\
\hline SBP CV & 8.14 & 1.19 to 55.5 & 0.032 & SBP CV & 1.49 & 0.35 to 6.25 & 0.588 \\
\hline SBP SV & 3.47 & 1.05 to 11.4 & 0.041 & SBP SV & 3.55 & 0.91 to 13.8 & 0.068 \\
\hline \multicolumn{4}{|c|}{ Good collaterals $(n=60)$} & \multicolumn{4}{|c|}{ Bad collaterals $(n=50)$} \\
\hline SBP SD & 5.78 & 1.23 to 27.2 & 0.027 & SBP SD & 1.85 & 0.60 to 5.74 & 0.289 \\
\hline SBP CV & 8.60 & 1.02 to 72.5 & 0.048 & SBP CV & 1.51 & 0.26 to 8.83 & 0.650 \\
\hline SBP SV & 3.82 & 1.15 to 12.7 & 0.029 & SBP SV & 2.09 & 0.58 to 7.47 & 0.258 \\
\hline
\end{tabular}

exhibit impaired cerebral autoregulation. ${ }^{10}{ }^{11}$ In patients with blunted autoregulation, increased BPV could produce deleterious fluctuations in cerebral perfusion, ${ }^{30}$ and would be particularly relevant in patients with large lesion, hypoperfused and mismatch volumes.

The detrimental effect of increased BPV was also seen in patients with PVO, which has been reported in previous studies, ${ }^{12} 31$ and in patients with good collaterals, which is a novel finding. Patients with PVO are more likely to have a large lesion core and hypoperfused volume, which could account for the differential effect. However, the susceptibility of patients with good collaterals was unexpected. Following ischaemic stroke, collateral blood vessels will dilate to provide additional blood flow $^{32}$ and patients with PVOs recruit more collateral vessels than those with distal occlusions. We propose that patients with PVO and good collaterals transmit the harmful increase in BPV to the area of the stroke, while those with worse collaterals or distal occlusions have a more isolated lesion core and ischaemic penumbra. The good collaterals could also expose the brain to cellular mediators of inflammation, which are elevated in patients with high BPV. ${ }^{33}{ }^{34}$ Finally, we cannot exclude other possible mechanisms such as cerebral oedema formation or other organ system damage resulting from increased BPV. ${ }^{15}$

This retrospective study has several limitations, including the non-uniform time intervals between BP measurements, time from stroke onset to first BP measurement and hospital discharge to clinical follow-up. Cataloguing use of BP-lowering or vasopressor medications was impractical given the many complexities in how patients were treated. The inclusion of only patients with CTP and angiographic imaging introduces the possibility of selection bias, although the baseline characteristics of our cohort were comparable to other studies of moderate-to-severe ischaemic stroke. We only included patients who had BP data for
120 hours after admission, but given the more severe strokes in our cohort and our ability to continue recording BP measurements if patients were transferred to the rehabilitation service, we do not feel this biased results.

\section{CONCLUSION}

$\mathrm{BPV}$ is a predictor of neurological outcome in patients with a large lesion core volume, concurrent viable ischaemic penumbra, PVO and good collaterals. Prior analyses of BPV have not accounted for perfusion imaging volumetric measurements or collateral status, rendering our findings novel and important for future BPV research in patients with acute ischaemic stroke. Dozens of clinical trials involving over 20000 patients have been conducted to determine if pharmacologically lowering BP after ischaemic stroke is beneficial. The results have been persistently neutral or negative. ${ }^{35-38}$ In contrast, there have been no clinical trials on the efficacy of reducing BPV after ischaemic stroke. Our study should help begin to clarify the inclusion criteria for such a trial. Furthermore, patients with ischaemic stroke who are not candidates for endovascular therapy (no Target Mismatch, low ASPECTS score from a large lesion core volume) or may not respond to intravenous tPA (PVOs recanalise in less than a quarter of patients administered $\mathrm{tPA})^{39}$ could specifically benefit from therapies aimed at reducing BPV, such as calcium channel blockers ${ }^{40}$ or low-dose vasopressors. ${ }^{41} 42$

Contributors AdH conceived of the study, reviewed all data, performed statistical analysis, and drafted and edited the manuscript. GJS assisted with statistical analysis. AB, LC and SO reviewed patient charts and performed assessment of imaging end points. JSM performed imaging assessment and edited the manuscript. JMM helped conceive the study, draft and edit the manuscript. GS and DT helped conceive the study and edit the manuscript.

Funding Research reported in this publication was supported by the National Center for Advancing Translational Sciences of the National Institutes of Health under Award Number KL2TR001065. 
Disclaimer The content is solely the responsibility of the authors and does not necessarily represent the official views of the National Institutes of Health.

Competing interests None declared.

Ethics approval The study was approved by the local Institutional Review Board and is retrospective research that did not involve a medical or surgical intervention.

Provenance and peer review Not commissioned; externally peer reviewed.

Data sharing statement No additional data are available.

Open Access This is an Open Access article distributed in accordance with the Creative Commons Attribution Non Commercial (CC BY-NC 4.0) license, which permits others to distribute, remix, adapt, build upon this work noncommercially, and license their derivative works on different terms, provided the original work is properly cited and the use is non-commercial. See: http:// creativecommons.org/licenses/by-nc/4.0/

\section{REFERENCES}

1. Geeganage C, Tracy M, England T, et al. for TAIST Investigators. Relationship between baseline blood pressure parameters (including mean pressure, pulse pressure, and variability) and early outcome after stroke: data from the Tinzaparin in Acute Ischaemic Stroke Trial (TAIST). Stroke 2011;42:491-3.

2. Sare GM, Ali M, Shuaib A, et al. Relationship between hyperacute blood pressure and outcome after ischemic stroke: data from the VISTA Collaboration. Stroke 2009;40:2098-103.

3. Chung JW, Kim N, Kang J, et al. Blood pressure variability and the development of early neurological deterioration following acute ischemic stroke. J Hypertens 2015;33:2099-106.

4. Manning L, Hirakawa $\mathrm{Y}$, Arima $\mathrm{H}$, et al. Blood pressure variability and outcome after acute intracerebral haemorrhage: a post-hoc analysis of INTERACT2, a randomised controlled trial. Lancet Neurol 2014:13:364-73.

5. Yong M, Kaste M. Association of characteristics of blood pressure profiles and stroke outcomes in the ECASS-II trial. Stroke 2008:39:366-72.

6. Endo K, Kario K, Koga M, et al. Impact of early blood pressure variability on stroke outcomes after thrombolysis: the SAMURAI rt-PA Registry. Stroke 2013;44:816-18.

7. Kang J, Ko Y, Park JH, et al. Effect of blood pressure on 3-month functional outcome in the subacute stage of ischemic stroke. Neurology 2012;79:2018-24

8. Paulson OB, Strandgaard S, Edvinsson L. Cerebral autoregulation. Cerebrovasc Brain Metab Rev 1990;2:161-92.

9. Jordan JD, Powers WJ. Cerebral autoregulation and acute ischemic stroke. Am J Hypertens 2012;25:946-50.

10. Derdeyn CP. Positron emission tomography imaging of cerebral ischemia. PET Clin 2007;2:35-44.

11. Powers WJ. Cerebral hemodynamics in ischemic cerebrovascular disease. Ann Neurol 1991;29:231-40.

12. Delgado-Mederos R, Ribo M, Rovira A, et al. Prognostic significance of blood pressure variability after thrombolysis in acute stroke. Neurology 2008;71:552-8

13. Campbell BC, Mitchell PJ, Kleinig TJ, et al., EXTEND-IA Investigators. Endovascular therapy for ischemic stroke with perfusion-imaging selection. N Engl J Med 2015;372:1009-18.

14. Luby M, Warach SJ, Albers GW, et al. Identification of imaging selection patterns in acute ischemic stroke patients and the influence on treatment and clinical trial enrollment decision making. Int J Stroke 2016;11:180-90.

15. Manning LS, Rothwell PM, Potter JF, et al. Prognostic significance of short-term blood pressure variability in acute stroke: systematic review. Stroke 2015;46:2482-90.

16. Gumbinger $C$, Gruschka $P$, Böttinger $M$, et al. Improved prediction of poor outcome after thrombolysis using conservative definitions of symptomatic hemorrhage. Stroke 2012;43:240-2.

17. French KF, Martinez JK, DeHavenon $\mathrm{AH}$, et al. Reproducibility of $\mathrm{ABC} / 2$ method to determine infarct volume and mismatch percentage with CT perfusion. J Neuroimaging 2014;24:232-7.
18. Maas MB, Lev MH, Ay H, et al. Collateral vessels on $\mathrm{CT}$ angiography predict outcome in acute ischemic. Stroke 2009;40:3001-5.

19. Nael K, Mossadeghi B, Boutelier T, et al. Bayesian estimation of cerebral perfusion using reduced-contrast-dose dynamic susceptibility contrast perfusion at 3T. Am J Neuroradiol 2014;36:710-8.

20. Kudo K, Boutelier T, Pautot $F$, et al. Bayesian analysis of perfusion-weighted imaging to predict infarct volume: comparison with singular value decomposition. Magn Reson Med Sci 2014;13:45-50.

21. Boutelier T, Kudo K, Pautot F, et al. Bayesian hemodynamic parameter estimation by bolus tracking perfusion weighted imaging IEEE Trans Med Imaging 2012;31:1381-95.

22. Wintermark M, Flanders AE, Velthuis B, et al. Perfusion-CT assessment of infarct core and penumbra receiver operating characteristic curve analysis in 130 patients suspected of acute hemispheric. Stroke 2006;37:979-85.

23. Alves JE, Carneiro Â, Xavier J. Reliability of CT perfusion in the evaluation of the ischaemic penumbra. Neuroradiol $J$ 2014;27:91-5.

24. Bivard A, Krishnamurthy V, Stanwell $P$, et al. Arterial spin labeling versus bolus-tracking perfusion in hyperacute stroke. Stroke J Cereb Circ 2014;45:127-33.

25. Lansberg MG, Straka M, Kemp S, et al. Magnetic resonance imaging profile and response to endovascular reperfusion: results of the DEFUSE 2 Prospective Cohort Study. Lancet Neurol 2012;11:860-7.

26. Saver JL, Gornbein J. Treatment effects for which shift or binary analyses are advantageous in acute stroke trials. Neurology 2009;72:1310-15.

27. Brown DL, Coffey CS. Stroke trials: a shift to shift analysis? Neurology 2009;72:1292-3.

28. Bath PMW, Lees KR, Schellinger PD, et al. Statistical analysis of the primary outcome in acute stroke trials. Stroke J Cereb Circ 2012;43:1171-8.

29. Ko Y, Park JH, Yang MH, et al. The significance of blood pressure variability for the development of hemorrhagic transformation in acute ischemic stroke. Stroke J Cereb Circ 2010:41:2512-

30. Rickards CA, Tzeng YC. Arterial pressure and cerebral blood flow variability: friend or foe? A review. Front Physiol 2014;5:120.

31. Buratti L, Cagnetti C, Balucani C, et al. Blood pressure variability and stroke outcome in patients with internal carotid artery occlusion. J Neurol Sci 2014;339:164-8.

32. Shuaib A, Butcher K, Mohammad AA, et al. Collateral blood vessels in acute ischaemic stroke: a potential therapeutic target. Lancet Neurol 2011;10:909-21.

33. Kim KI, Lee JH, Chang HJ, et al. Association between blood pressure variability and inflammatory marker in hypertensive patients. Circ J 2008;72:293-8.

34. Tatasciore A, Zimarino M, Renda G, et al. Awake blood pressure variability, inflammatory markers and target organ damage in newly diagnosed hypertension. Hypertens Res 2008;31:2137-46.

35. Geeganage C, Bath PM. Vasoactive drugs for acute stroke. Cochrane Database Syst Rev 2010;(7):CD002839.

36. Robinson TG, Potter JF, Ford GA, et al. Effects of antihypertensive treatment after acute stroke in the Continue or Stop Post-Stroke Antihypertensives Collaborative Study (COSSACS): a prospective, randomised, open, blinded-endpoint trial. Lancet Neurol 2010;9:767-75.

37. Sandset EC, Murray GD, Bath PMW, et al. Relation between change in blood pressure in acute stroke and risk of early adverse events and poor outcome. Stroke 2012;43:2108-14.

38. Wang $\mathrm{H}$, Tang $\mathrm{Y}$, Rong $\mathrm{X}$, et al. Effects of early blood pressure lowering on early and long-term outcomes after acute stroke: an updated meta-analysis. PLOS ONE 2014;9:e97917.

39. Bhatia $\mathrm{R}$, Hill MD, Shobha $\mathrm{N}$, et al. Low rates of acute recanalization with intravenous recombinant tissue plasminogen activator in ischemic stroke: real-world experience and a call for action. Stroke J Cereb Circ 2010;41:2254-8.

40. Rothwell PM, Howard SC, Dolan E, et al. Effects of $\beta$ blockers and calcium-channel blockers on within-individual variability in blood pressure and risk of stroke. Lancet Neurol 2010;9:469-80.

41. Sherwood A, Steffen PR, Blumenthal JA, et al. Nighttime blood pressure dipping: the role of the sympathetic nervous system. $A m$ $J$ Hypertens 2002;15:111-18.

42. Conway J, Boon N, Vann Jones J, et al. Mechanisms concerned with blood pressure variability throughout the day. Clin Exp Hypertens A 1985;7:153-7. 